\title{
Modeling and control of the vibration of two beams coupled with fluid and active links
}

\author{
Zhiyi Zhang*, Fang Hu, Zeng Li and Hongxing Hua \\ State Key Laboratory of Mechanical Systems and Vibration, Shanghai Jiaotong University, Shanghai, China
}

Received 23 November 2010

Revised 28 July 2011

\begin{abstract}
Investigated are modeling and control approaches for vibration analysis of two identical beams which are coupled with fluid and active mechanical links. In the modeling of the coupled beam system, orthogonal functions are used to represent vibration of the beams and the fluid-structure interaction is considered. Frequency Response Functions (FRFs) are derived from the coupled governing equations and the superposition principle for linear vibration systems. In the control of vibration of the beams, impulse response functions corresponding to the FRFs and an adaptive control algorithm are employed to attenuate vibration transmission between the two beams. Natural frequencies, mode shapes as well as the pressure distribution in the fluid are computed. The results obtained by the proposed modeling method are in good consistency with those obtained by the finite element analysis. Moreover, it is demonstrated that the active mechanical link is able to reduce vibration transmission and change the deformation of beams as well as the distribution of fluid pressure.
\end{abstract}

Keywords: Fluid-structure interaction, modal analysis, frequency response function, active control

\section{Introduction}

The bounded fluid between double-layer shells, plates or beams can transfer vibrations from one side to the other and couples the motion of structures. Apart from the bounded fluid, mechanical links between shells, plates or beams affect vibration transmission as well. However, vibration transmission contributed by the fluid and the mechanical links is of different mechanism. For the purpose of vibration control, the mechanical links can be embedded with active elements to generate counteracting vibrations. Hence, when one side of the structure is excited by disturbance forces, reduction of vibration at the other side can be expected. However, investigation on the mechanism of reducing vibration transmission in double-layer shells, plates or beams through embedded actuators is insufficient. This study attempts to analyze the coupled vibration in such systems by using a simplified physical model, which consists of two identical beams, a bounded heavy fluid layer and a mechanical link. Although this model is relatively simple, it is able to reveal vibration characteristics and their evolution under active control. Moreover, fluid-structure interaction is involved in it, which makes the modeling and control of vibration transmission become a difficult problem.

Interaction between structures and the fluid can greatly affect the dynamic behavior of structures. The research on fluid-structure interaction (FSI) started at least in the early 1950s, and the early methods are mainly analytical ones which deal with FSI in those systems of simple construction [1-3]. With the development of computation technology, numerical methods, such as FEM and FEM/BEM, are widely used in theoretical investigation as well as engineering applications. However, analytical methods are still preferable in the investigation of FSI and control of simple systems, which can give general but important understanding to the coupled dynamic behavior. There is some research about the coupled vibration of beam structures and the fluid, such as the analyses of vibration of cantilever

\footnotetext{
*Corresponding author. E-mail: chychang@ sjtu.edu.cn.
} 


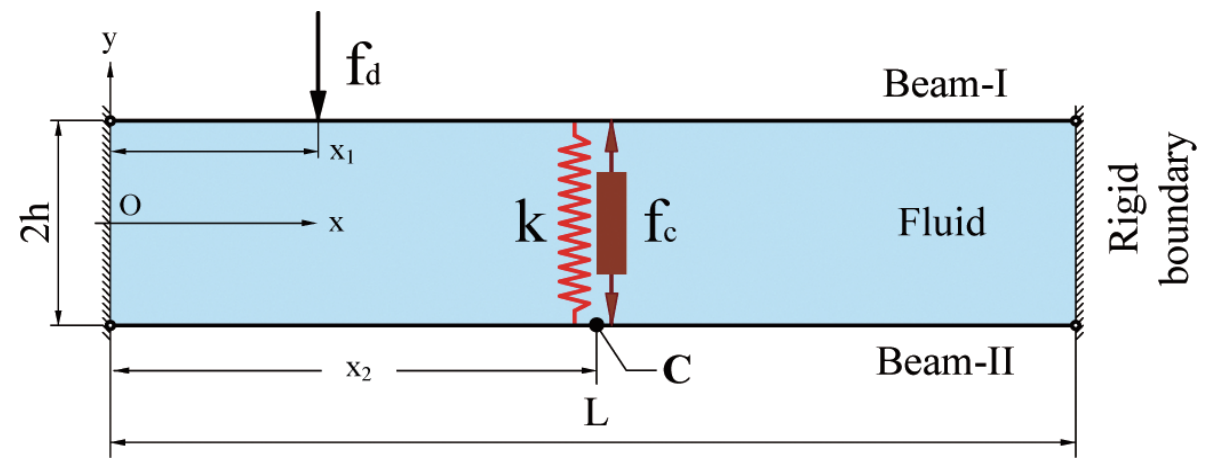

Fig. 1. Physical model of the double-beam structure.

beams immersed wholly or partly in water [4-6], where the wave equation and different boundary conditions are considered for the infinite fluid domain. Moreover, there are also problems involving structures and bounded fluid and their interaction [7], for example, a beam structure floating on the bounded fluid and its response induced by a moving load are analyzed by Fleischer and Park [8]. The analytical methods for coupled vibration equations usually adopt modal analysis and Fourier series and hence yield accurate solutions. However, approximation in the solution of FSI can also give good results in some cases [9]. In FSI problems, the added mass effect of fluid is important to structural responses and some models are proposed to take into account this effect in the estimation of natural vibration frequencies [10-13]. Computation of vibration of double-walled panels with bounded fluid and mechanical links has been studied, but most investigations deal with interaction between structures and a light fluid, such as the air [14-16]. In this study, the involved fluid is water and the boundary conditions are totally different, which in fact demands a new approach of modeling.

On the control of vibration of mechanical systems, there is lots of research contributing to the methods of modeling and control as well as the implementation of control systems [17-20]. For the frequency-domain methods, FRFs are necessary for the construction of controllers. Even for some time-domain methods, FRFs are still needed to obtain time series or state space models. Modeling of FSI usually results in a frequency-domain model, therefore, in this study, the modeling is completed in the frequency domain and the derived FRF model is subsequently transformed into a time domain model. Active control forces are then computed on the basis of this time-domain model.

The following discussion is organized in six sections. Mathematical description of the coupled vibration is given in Section 2, and the solution to these equations is presented in Section 3. Computation of FRFs and the adaptive control are given respectively in Section 4 and Section 5. Simulation results about the modeling and control are discussed in Section 6, and finally the conclusions are made in Section 7.

\section{Mathematical description}

The physical model is shown in Fig. 1, where two beams are simply supported and connected by a spring and an actuator at the center of the beams. The beams are a plate segment of unit width, cut from a semi-infinite plate model with distributed elastic links and under the action of line forces. This type of simplification degrades a three dimensional problem to a two dimensional one and it is for theoretical analysis only. For the simplicity of discussion, the two beams are assumed to be identical, having the same mechanical properties and dimensions. The length of the beams is $L$, the distance between the beams is $2 \mathrm{~h}$, the stiffness of the spring is $k$ and the side walls are rigid boundaries. Further assumptions on the fluid and the beams include: (1) The system is in small vibration; (2) The fluid is inviscid and compressible; (3) no gravity is present; (4) The material is isotropic and of linear elasticity; (5) Shear deformation and rotary inertia of the beams are negligible. Hence, the motion equation for the fluid is [21]

$$
\frac{\partial^{2} p}{\partial x^{2}}+\frac{\partial^{2} p}{\partial y^{2}}=\frac{1}{c^{2}} \frac{\partial^{2} p}{\partial t^{2}}
$$


where $p$ is the sound pressure and $c$ the speed of sound in the fluid. Let $p(x, y, t)=X(x) Y(y) e^{j \omega t}$, then from Eq. (1), one has

$$
X^{\prime \prime}(x)+k_{x}^{2} X(x)=0, \quad Y^{\prime \prime}(y)+k_{y}^{2} Y(y)=0
$$

where $j=\sqrt{-1}, k_{x}^{2}+k_{y}^{2}=(\omega / c)^{2}, \omega$ is the circular frequency. The boundary conditions at $x=0$ and $x=L$ are

$$
\left.\frac{\partial p}{\partial x}\right|_{x=0}=0,\left.\quad \frac{\partial p}{\partial x}\right|_{x=L}=0
$$

Therefore,

$$
X_{n}(x)=\cos k_{x n} x, \quad k_{x n}=\frac{n \pi}{L}, \quad n=1,2, \cdots
$$

When $k_{y}^{2}=(\omega / c)^{2}-k_{x}^{2}<0, k_{y}$ is an imaginary value. Let $k_{y}=j k_{y}^{\prime}$, one can have $k_{y}^{\prime}=\sqrt{(n \pi / L)^{2}-(\omega / c)^{2}}$, $n=1,2, \cdots$, and

$$
Y_{n}(y)=C_{n} \sinh \left(k_{y n}^{\prime} y\right)+D_{n} \cosh \left(k_{y n}^{\prime} y\right), \quad n=1,2, \cdots
$$

where $C_{n}$ and $D_{n}$ are complex coefficients. Combining Eqs (4) and (5), the sound pressure can be given as

$$
p(x, y, t)=e^{j \omega t} \sum_{n=1}^{\infty}\left(C_{n} \sinh \left(k_{y n}^{\prime} y\right)+D_{n} \cosh \left(k_{y n}^{\prime} y\right)\right) \cos \left(k_{x n} x\right)
$$

The equations for transverse vibration of the beams are

$$
\begin{aligned}
\text { Beam - I : } & E I \frac{\partial^{4} \eta_{1}(x, t)}{\partial x^{4}}+m \frac{\partial^{2} \eta_{1}(x, t)}{\partial t^{2}}+k\left(\eta_{1}\left(x_{2}, t\right)-\eta_{2}\left(x_{2}, t\right)\right) \delta\left(x-x_{2}\right) \\
& =p(x, h, t)+F_{0} \delta\left(x-x_{1}\right) e^{j \omega t} \\
\text { Beam - II }: & E I \frac{\partial^{4} \eta_{2}(x, t)}{\partial x^{4}}+m \frac{\partial^{2} \eta_{2}(x, t)}{\partial t^{2}}-k\left(\eta_{1}\left(x_{2}, t\right)-\eta_{2}\left(x_{2}, t\right)\right) \delta\left(x-x_{2}\right)=-p(x,-h, t)
\end{aligned}
$$

where $E I$ is the bending stiffness, $m$ the mass density of the beam of unit length, $k$ the stiffness of the spring, $\delta(\cdot)$ the Dirac delta function, $x_{1}$ the coordinate of the point where the excitation force acts, $F_{0}$ the amplitude of the force, $x_{2}$ the coordinate of the point where the spring and the actuator are mounted, $\eta_{i}(x, t)(i=1,2)$ are the transverse displacements of Beam-I and Beam-II, respectively, and $\eta_{i}(x, t)=\bar{\eta}_{i}(x) e^{j \omega t}$, the directions of $\eta_{i}(x, t)$ coincide with those of the global coordinates. On the interfaces where the fluid and the beams are coupled, the coupling conditions are given as

$$
\left.\frac{\partial p(x, y, t)}{\partial y}\right|_{y=h}=\rho \omega^{2} \eta_{1}(x, t),\left.\quad \frac{\partial p(x, y, t)}{\partial y}\right|_{y=-h}=\rho \omega^{2} \eta_{2}(x, t)
$$

where $\rho$ is the mass density of the fluid. Substituting Eq. (6) into Eq. (9) and multiplying both sides of Eq. (9) by $\cos k_{x n} x$ and then integrating both sides on $[0, L], C_{n}, D_{n}$ can be given as follows:

$$
C_{n}=\frac{\rho \omega^{2} \int_{0}^{L}\left(\bar{\eta}_{1}(x)+\bar{\eta}_{2}(x)\right) \cos \left(k_{x n} x\right) d x}{L k_{y n}^{\prime} \cosh \left(k_{y n}^{\prime} h\right)}, \quad D_{n}=\frac{\rho \omega^{2} \int_{0}^{L}\left(\bar{\eta}_{1}(x)-\bar{\eta}_{2}(x)\right) \cos \left(k_{x n} x\right) d x}{L k_{y n}^{\prime} \sinh \left(k_{y n}^{\prime} h\right)}
$$

Therefore, Eq. (6) can be rewritten as

$$
\begin{aligned}
p(x, y, t) & =e^{j \omega t} \frac{\rho \omega^{2}}{L} \sum_{n=1}^{\infty} \frac{1}{k_{y n}^{\prime}}\left(\frac{\int_{0}^{L}\left(\bar{\eta}_{1}(x)+\bar{\eta}_{2}(x)\right) \cos k_{x n} x d x}{\cosh k_{y n}^{\prime} h} \sinh k_{y n}^{\prime} y\right. \\
& \left.+\frac{\int_{0}^{L}\left(\bar{\eta}_{1}(x)-\bar{\eta}_{2}(x)\right) \cos k_{x n} x d x}{\sinh k_{y n}^{\prime} h} \cosh k_{y n}^{\prime} y\right) \cos k_{x n} x
\end{aligned}
$$

Since the beams are simply supported, there exists 


$$
\eta_{i}(0, t)=\eta_{i}(L, t)=0,\left.\quad \frac{\partial^{2} \eta_{i}(x, t)}{\partial x^{2}}\right|_{x=0}=\left.\frac{\partial^{2} \eta_{i}(x, t)}{\partial x^{2}}\right|_{x=L}=0, \quad i=1,2
$$

Moreover, the fluid needs to satisfy the condition that the total mass keeps invariable, and accordingly

$$
\int_{0}^{L} 2 h \rho\left(\eta_{1}(x, t)-\eta_{2}(x, t)\right) d x=0
$$

Equation (13) implies that the displacements of the two beams are not independent.

\section{Computation of the coupled vibration}

Suppose $x_{2}=L / 2$, vibration modes of the beams can be divided into the symmetric and anti-symmetric ones. In the following discussion, computation of coupled vibration is presented according to these two categories of modes.

\subsection{Computation of anti-symmetric modes}

First, consider the response composed of the anti-symmetric modes only. In this case, the amplitude of displacement of each beam can be described easily as

$$
\bar{\eta}_{i}(x)=\sum_{r=2,4 \ldots}^{\infty} A_{i r} \varphi_{r}(x), \quad i=1,2
$$

where $\varphi_{r}(x)=\sqrt{\frac{2}{m L}} \sin \frac{r \pi x}{L}(r=2,4 \ldots)$ are the expansion functions that satisfy the conditions given by Eq. (12) and $m$ is the mass density of the beam. Substituting Eq. (14) into Eq. (11), Eqs (7) and (8), then multiplying both sides of Eqs (7) and (8) by $\varphi_{s}(x)$ and integrating both sides on $[0, L]$, one can obtain

$$
\begin{aligned}
& A_{1 r}\left(\omega_{r}^{2}-\omega^{2}\right)+k \sum_{s=2,4 \ldots}^{\infty}\left(A_{1 s}-A_{2 s}\right) \varphi_{s}\left(x_{2}\right) \varphi_{r}\left(x_{2}\right) \\
& =\rho \omega^{2} \sum_{n=1}^{\infty}\left(\frac{I_{n r}}{L k_{y n}^{\prime}}\left(\tanh \left(k_{y n}^{\prime} h\right) \sum_{s=2,4 \ldots}^{\infty}\left(A_{1 s}+A_{2 s}\right) I_{n s}+\operatorname{coth}\left(k_{y n}^{\prime} h\right) \sum_{s=2,4 \ldots}^{\infty}\left(A_{1 s}-A_{2 s}\right) I_{n s}\right)\right) \\
& +F_{0} \varphi_{r}\left(x_{1}\right) \\
& A_{2 r}\left(\omega_{r}^{2}-\omega^{2}\right)-k \sum_{s=2,4 \ldots}^{\infty}\left(A_{1 s}-A_{2 s}\right) \varphi_{s}\left(x_{2}\right) \varphi_{r}\left(x_{2}\right) \\
& =\rho \omega^{2} \sum_{n=1}^{\infty}\left(\frac { I _ { n r } } { L k _ { y n } ^ { \prime } } \left(\left[\tanh \left(k_{y n}^{\prime} h\right)+\operatorname{coth}\left(k_{y n}^{\prime} h\right)\right] \sum_{s=2,4 \ldots}^{\infty} A_{2 s} I_{n s}+\left[\tanh \left(k_{y n}^{\prime} h\right)\right.\right.\right. \\
& \left.\left.\left.-\operatorname{coth}\left(k_{y n}^{\prime} h\right)\right] \sum_{s=2,4 \ldots}^{\infty} A_{1 s} I_{n s}\right)\right)
\end{aligned}
$$

where

$$
I_{n r}=\int_{0}^{L} \cos \left(k_{x n} x\right) \varphi_{r}(x) d x=\left\{\begin{array}{cc}
\sqrt{\frac{2}{m L}} \frac{2 r}{\pi\left(r^{2}-n^{2}\right)} & n=1,3 \ldots \\
0 & n=2,4 \ldots
\end{array}\right\}, \omega_{r}=\sqrt{\frac{E I}{m}}\left(\frac{r \pi}{L}\right)^{2}, r=2,4 \ldots
$$

Defining

$$
\alpha_{n}=\frac{1}{L k_{y n}^{\prime}}\left[\tanh \left(k_{y n}^{\prime} h\right)+\operatorname{coth}\left(k_{y n}^{\prime} h\right)\right], \quad \beta_{n}=\frac{1}{L k_{y n}^{\prime}}\left[\tanh \left(k_{y n}^{\prime} h\right)-\operatorname{coth}\left(k_{y n}^{\prime} h\right)\right],
$$


and using finite terms to approximate Eqs (15) and (16), one can obtain a linear matrix equation:

$$
\left[\begin{array}{cc}
\mathbf{S} & -\mathbf{T} \\
-\mathbf{T} & \mathbf{S}
\end{array}\right]\left[\begin{array}{l}
\mathbf{A}_{1} \\
\mathbf{A}_{2}
\end{array}\right]=\left[\begin{array}{l}
\mathbf{F} \\
\mathbf{0}
\end{array}\right]
$$

where

$$
\begin{aligned}
& \mathbf{S}=[\mathbf{W}]-\omega^{2}\left([\mathbf{E}]+\rho[\mathbf{I}]^{T}[\alpha][\mathbf{I}]\right)+k[\Phi][\Phi]^{T}, \quad \mathbf{T}=k[\Phi][\Phi]^{T}+\rho \omega^{2}[\mathbf{I}]^{T}[\beta][\mathbf{I}], \\
& {[\alpha]=\operatorname{diag}\left(\begin{array}{llll}
\alpha_{1} & \alpha_{3} & \cdots & \alpha_{N}
\end{array}\right), \quad[\beta]=\operatorname{diag}\left(\beta_{1}, \quad \beta_{3}, \quad \cdots, \quad \beta_{N}\right),} \\
& {[\Phi]=\left[\begin{array}{llll}
\varphi_{2}\left(x_{2}\right), & \varphi_{4}\left(x_{2}\right), \quad \ldots, \quad \varphi_{R}\left(x_{2}\right)
\end{array}\right]^{T}, \quad[\mathbf{W}]=\operatorname{diag}\left(\omega_{2}^{2}, \quad \omega_{4}^{2}, \quad \cdots, \quad \omega_{R}^{2}\right),} \\
& {[\mathbf{F}]=F_{0}\left[\varphi_{2}\left(x_{1}\right), \quad \varphi_{4}\left(x_{1}\right), \quad \ldots, \quad \varphi_{R}\left(x_{1}\right)\right]^{T}, \quad\left[\mathrm{~A}_{i}\right]=\left[\begin{array}{llll}
A_{i 2}, & A_{i 4}, & \ldots, & A_{i R}
\end{array}\right]^{T},}
\end{aligned}
$$

$i=1,2 \ldots,[\mathbf{E}]$ is the identity matrix, $[\mathbf{I}]=\left[\mathbf{I}_{n r}\right]_{N \times R}$, the subscript $\mathrm{N}$ is the number of retained terms in the expansion of sound pressure, and $\mathrm{R}$ the number of retained terms in the expansion of displacement of the beam.

Solving Eq. (17) will give $A_{i r}(i=1,2$ and $r=2,4, \ldots)$, and the amplitude of each beam is computed by

$$
\bar{\eta}_{i}(x)=[\varphi]\left[\mathbf{A}_{i}\right], \quad[\varphi]=\left[\phi_{2}(x), \phi_{4}(x), \ldots, \phi_{R}(x)\right], \quad i=1,2
$$

The distribution of sound pressure is given approximately by

$$
\begin{aligned}
p(x, y) & =\frac{\rho \omega^{2}}{L} \sum_{n=1,3 \ldots}^{N} \frac{1}{k_{y n}^{\prime}}\left(\frac{\sinh \left(k_{y n}^{\prime} y\right)}{\cosh \left(k_{y n}^{\prime} h\right)} \sum_{r=2,4 \ldots}^{R}\left(A_{1 r}+A_{2 r}\right) I_{n r}\right. \\
& \left.+\frac{\cosh \left(k_{y n}^{\prime} y\right)}{\sinh \left(k_{y n}^{\prime} h\right)} \sum_{r=2,4 \ldots}^{R}\left(A_{1 r}-A_{2 r}\right) I_{n r}\right) \cos \left(k_{x n} x\right)
\end{aligned}
$$

\subsection{Computation of symmetric modes}

Next, consider the response composed of symmetric modes only. In this case, mode shapes cannot be computed in a way similar to Eq. (14). However, the amplitude of displacement of each beam can be expressed in Fourier series

$$
\bar{\eta}_{i}(x)=\frac{B_{i 0}^{(0)}}{2}+\sum_{r=2,4 \ldots}^{\infty} B_{i r}^{(0)} \cos \frac{r \pi x}{L}, \quad i=1,2
$$

The conditions given by Eq. (13) results in

$$
B_{i 0}^{(0)}=0
$$

Similarly, the derivatives of $\bar{\eta}_{i}(x)$ are expanded as well in Fourier series

$$
\eta_{i}^{(k)}(x)=\frac{B_{i 0}^{(k)}}{2}+\sum_{r=1}^{\infty} B_{i r}^{(k)} \cos \frac{r \pi x}{L}, \quad k=1,2,3,4
$$

where the superscript $k$ in $\eta_{i}^{(k)}(x)$ denotes the kth derivative of $\bar{\eta}_{i}(x)$. According to the boundary conditions given by Eqs (12) and (22), one can obtain the following equations [8]:

$$
\begin{aligned}
& B_{i 0}^{(0)}=\frac{B_{i 0}^{(4)}}{120}-\frac{2}{\pi^{4}} \sum_{s=2,4 \ldots}^{\infty} \frac{B_{i s}^{(4)}}{s^{2}}\left(\frac{\pi^{2}}{12}+\frac{1}{s^{2}}\right) \\
& B_{i r}^{(0)}=-\frac{B_{i 0}^{(4)}}{\pi^{4} r^{2}}\left(\frac{\pi^{2}}{12}+\frac{1}{r^{2}}\right)+\frac{2}{\pi^{4} r^{2}} \sum_{s=2,4 \ldots}^{\infty} \frac{B_{i s}^{(4)}}{s^{2}}+\frac{B_{i r}^{(4)}}{\pi^{4} r^{4}}, r=2,4, \cdots
\end{aligned}
$$

From Eqs (21) and (23), one has 


$$
B_{i 0}^{(4)}=\frac{240}{\pi^{4}} \sum_{r=2,4 \ldots}^{\infty} \frac{B_{i r}^{(4)}}{r^{2}}\left(\frac{\pi^{2}}{12}+\frac{1}{r^{2}}\right)
$$

Substituting Eqs (20) and (22) into Eqs (7) and (8), then multiplying both sides of Eqs (7)-(8) by $\cos \frac{r \pi x}{L}(r=$ $2,4, \ldots)$ and integrating both sides on $[0, \mathrm{~L}]$, one can obtain

$$
\begin{aligned}
\frac{E I L}{2} B_{1 r}^{(4)} & -\frac{m \omega^{2} L}{2} B_{1 r}^{(0)}+k\left(\sum_{s=2,4 \ldots}^{\infty} B_{1 s}^{(0)} \cos \frac{s \pi x_{2}}{L}-\sum_{s=2,4 \ldots}^{\infty} B_{2 s}^{(0)} \cos \frac{s \pi x_{2}}{L}\right) \cos \frac{r \pi x_{2}}{L} \\
& =\int_{0}^{L} p(x, h, 0) \cos \frac{r \pi x}{L} d x+F_{0} \cos \frac{r \pi x_{1}}{L} \\
\frac{E I L}{2} B_{2 r}^{(4)} & -\frac{m \omega^{2} L}{2} B_{2 r}^{(0)}-k\left(\sum_{s=2,4 \ldots}^{\infty} B_{1 s}^{(0)} \cos \frac{s \pi x_{2}}{L}-\sum_{s=2,4 \ldots}^{\infty} B_{2 s}^{(0)} \cos \frac{s \pi x_{2}}{L}\right) \cos \frac{r \pi x_{2}}{L} \\
& =-\int_{0}^{L} p(x,-h, 0) \cos \frac{r \pi x}{L} d x
\end{aligned}
$$

Substituting Eqs (11) and (24)-(25) into Eqs (26)-(27) and retaining finite terms, one can obtain

$$
\left[\begin{array}{cc}
{\left[\Lambda_{1}\right]-[\sigma]+[\Theta]} & -\left(\left[\Lambda_{2}\right]+[\tau]+[\Theta]\right) \\
-\left(\left[\Lambda_{2}\right]+[\tau]+[\Theta]\right) & {\left[\Lambda_{1}\right]-[\sigma]+[\Theta]}
\end{array}\right]\left[\begin{array}{l}
\mathrm{B}_{1} \\
\mathrm{~B}_{2}
\end{array}\right]=\left[\begin{array}{c}
\mathrm{F} \\
0
\end{array}\right]
$$

where

$$
\begin{aligned}
& {\left[\Lambda_{1}\right]=\operatorname{diag}\left(E I L-\frac{\omega^{2}\left(m L+\rho \alpha_{2}\right)}{(2 \pi)^{4}}, E I L-\frac{\omega^{2}\left(m L+\rho \alpha_{4}\right)}{(4 \pi)^{4}}, \cdots, E I L-\frac{\omega^{2}\left(m L+\rho \alpha_{R}\right)}{(R \pi)^{4}}\right),} \\
& {\left[\Lambda_{2}\right]=\operatorname{diag}\left(\frac{\rho \omega^{2} \beta_{2}}{(2 \pi)^{4}}, \frac{\rho \omega^{2} \beta_{4}}{(4 \pi)^{4}}, \cdots, \frac{\rho \omega^{2} \beta_{R}}{(R \pi)^{4}}\right),}
\end{aligned}
$$

$R$ is a positive even number,

$$
\begin{aligned}
& {[\xi]=\left[\xi_{2}, \xi_{4}, \cdots, \xi_{R}\right]^{T}, \quad[\zeta]=\left[\zeta_{2}, \zeta_{4}, \cdots, \zeta_{R}\right]^{T}, \quad \xi_{s}=-\frac{1}{s^{2}}\left(\frac{\pi^{2}}{12}+\frac{1}{s^{2}}\right), \quad \zeta_{s}=\frac{1}{s^{2}}, \quad s=2,4, \cdots,} \\
& \Sigma_{1}=\sum_{s=2,4 \ldots}^{R}-\frac{240}{\pi^{8}} \xi_{s} \cos \frac{s \pi x_{2}}{L}, \quad \Sigma_{2}=\sum_{s=2,4 \ldots}^{R}-\frac{240}{\pi^{8}} \zeta_{s} \cos \frac{s \pi x_{2}}{L}, \\
& {[\Phi]=\left[\cos \frac{2 \pi x_{2}}{L}, \cos \frac{4 \pi x_{2}}{L}, \ldots, \cos \frac{R \pi x_{2}}{L}\right]^{T}, \quad v_{r s}=\frac{1}{\pi^{4} s^{4}} \cos \frac{r \pi x_{2}}{L} \cos \frac{s \pi x_{2}}{L}, \quad r=2,4, \cdots, s=2,4, \cdots} \\
& \sigma_{r s}=\left(m L+\rho \alpha_{r}\right) \omega^{2}\left(-\frac{240}{\pi^{8}} \xi_{r} \xi_{s}+\frac{2}{\pi^{4}} \zeta_{r} \zeta_{s}\right), \quad \tau_{r s}=\rho \omega^{2} \beta_{r}\left(-\frac{240}{\pi^{8}} \xi_{r} \xi_{s}+\frac{2}{\pi^{4}} \zeta_{r} \zeta_{s}\right), \\
& {[\mathrm{F}]=2 F_{0}\left[\cos \frac{2 \pi x_{1}}{L}, \cos \frac{4 \pi x_{1}}{L}, \cdots, \cos \frac{R \pi x_{1}}{L}\right]^{T}, \quad\left[\mathrm{~B}_{1}\right]=\left[B_{12}^{(4)}, B_{14}^{(4)}, \cdots, B_{1 R}^{(4)}\right]^{T},} \\
& {\left[\mathrm{~B}_{2}\right]=\left[B_{22}^{(4)}, B_{24}^{(4)}, \cdots, B_{2 R}^{(4)}\right]^{T}, \quad[\Theta]=2 k\left(\Sigma_{1}[\Phi][\xi]^{T}+\Sigma_{2}[\Phi][\zeta]^{T}+[v]\right) .}
\end{aligned}
$$

Solving Eq. (28) will give $B_{i r}^{(4)}(i=1,2$ and $r=2,4, \ldots, R)$ and further lead to

$$
B_{i r}^{(0)}=-\frac{240}{\pi^{8} r^{2}}\left(\frac{\pi^{2}}{12}+\frac{1}{r^{2}}\right) \sum_{s=2,4 \ldots}^{R} \frac{B_{i s}^{(4)}}{s^{2}}\left(\frac{\pi^{2}}{12}+\frac{1}{s^{2}}\right)+\frac{2}{\pi^{4} r^{2}} \sum_{s=2,4 \ldots}^{R} \frac{B_{i s}^{(4)}}{s^{2}}+\frac{B_{i r}^{(4)}}{\pi^{4} r^{4}}
$$

According to Eqs (29), (20) and (11), one can obtain the symmetric natural modes as well as the corresponding pressure distribution. 


\subsection{Computation of the first mode}

It should be noted that the first natural mode, i.e. $\eta_{1}(x, t)=\eta_{2}(x, t)$ and without middle nodes, cannot be obtained from Eq. (14) or Eq. (20). However, there are approximate methods which can be applied to derive the first natural mode [11-13]. Taking the fluid as added mass to the beams and representing the first vibration mode of the beams by

$$
\varphi(x)=a \sin \frac{\pi x}{L}
$$

the equation for the first modal vibration can thus be given by

$$
E I \frac{\partial^{4} \eta(x, t)}{\partial x^{4}}+(m+h \rho) \frac{\partial^{2} \eta(x, t)}{\partial t^{2}}=F_{0} \delta\left(x-x_{1}\right) e^{j \omega t}
$$

where $\eta=\eta_{1}=\eta_{2}=\varphi(x) e^{j \omega t}$. From Eq. (31), one can obtain the first natural frequency.

\section{FRF and IRF}

Vibration responses of the beams are composed of the symmetric and anti-symmetric modes, and the frequency response functions (FRFs) can be synthesized from these modes according to the superposition principle. In fact, Eqs (7) and (8) can be recast as

$$
\begin{gathered}
\left\{\left[\begin{array}{cc}
E I & 0 \\
0 & E I
\end{array}\right] \frac{\partial^{4}}{\partial x^{4}}+\left[\begin{array}{cc}
m & 0 \\
0 & m
\end{array}\right] \frac{\partial^{2}}{\partial t^{2}}+\left[\begin{array}{cc}
k \delta\left(x-x_{2}\right) & -k \delta\left(x-x_{2}\right) \\
-k \delta\left(x-x_{2}\right) & k \delta\left(x-x_{2}\right)
\end{array}\right]\right\}\left\{\begin{array}{l}
\eta_{1} \\
\eta_{2}
\end{array}\right\}-\left\{\begin{array}{l}
p_{1} \\
p_{2}
\end{array}\right\} \\
=\left\{\begin{array}{c}
F_{0} \delta\left(x-x_{1}\right) \delta(t) \\
0
\end{array}\right\}
\end{gathered}
$$

where $p_{1}=p(x, h, t), p_{2}=-p(x,-h, t)$. The Fourier transform of Eq. (32) is

$$
\left\{\mathrm{K} \frac{\partial^{4}}{\partial x^{4}}-\mathrm{M} \omega^{2}+\mathrm{K}_{12}\right\} \bar{\eta}-\overline{\mathrm{p}}=\mathrm{F} \delta\left(x-x_{1}\right)
$$

where

$$
\begin{array}{ll}
\mathrm{K}=\left[\begin{array}{cc}
E I & 0 \\
0 & E I
\end{array}\right], \quad \mathrm{M}=\left[\begin{array}{cc}
m & 0 \\
0 & m
\end{array}\right], \quad \mathrm{K}_{12}=\left[\begin{array}{cc}
k \delta\left(x-x_{2}\right) & -k \delta\left(x-x_{2}\right) \\
-k \delta\left(x-x_{2}\right) & k \delta\left(x-x_{2}\right)
\end{array}\right] \\
\bar{\eta}=\int_{-\infty}^{+\infty} \eta e^{-j \omega t} d t, \quad \overline{\mathrm{p}}=\int_{-\infty}^{+\infty} \mathrm{p} e^{-j \omega t} d t, \quad \eta=\left\{\begin{array}{l}
\eta_{1} \\
\eta_{2}
\end{array}\right\}, \quad \mathrm{p}=\left\{\begin{array}{l}
p_{1} \\
p_{2}
\end{array}\right\}, \quad \mathrm{F}=\left\{\begin{array}{c}
F_{0} \\
0
\end{array}\right\} .
\end{array}
$$

According to Eqs (33) and (11), for the natural response $\eta_{u}$ at $\omega_{u}$, there exists

$$
\left\{\mathrm{K} \frac{\partial^{4}}{\partial x^{4}}-\mathbf{M} \omega^{2}+\mathrm{K}_{12}\right\} \bar{\eta}_{u}-\overline{\mathrm{p}}_{u}=0, \quad \overline{\mathrm{p}}_{u}=\rho \omega_{u}^{2} \sum_{n}\left[\begin{array}{c}
\alpha_{n} \beta_{n} \\
\beta_{n} \alpha_{n}
\end{array}\right] \cos k_{x n} x \int_{0}^{L} \bar{\eta}_{u} \cos k_{x n} x d x
$$

where $\bar{\eta}_{u}=\int_{-\infty}^{+\infty} \eta_{u} e^{-j \omega t} d t, \overline{\mathrm{p}}_{u}=\int_{-\infty}^{+\infty} \mathrm{p}_{u} e^{-j \omega t} d t$. Equation (34) further implies

$$
\int_{0}^{L} \bar{\eta}_{v}^{T}\left(\left\{\mathrm{~K} \frac{\partial^{4}}{\partial x^{4}}-\mathrm{M} \omega_{u}^{2}+\mathrm{K}_{12}\right\} \bar{\eta}_{u}-\overline{\mathrm{p}}_{u}\right) d x=0, \int_{0}^{L} \bar{\eta}_{u}^{T}\left(\left\{\mathrm{~K} \frac{\partial^{4}}{\partial x^{4}}-\mathbf{M} \omega_{v}^{2}+\mathrm{K}_{12}\right\} \bar{\eta}_{v}-\overline{\mathrm{p}}_{v}\right) d x=0
$$

where $\eta_{v}$ is the response of a natural vibration mode at $\omega_{v}$, and 


$$
\bar{\eta}_{v}=\int_{-\infty}^{+\infty} \eta_{v} e^{-j \omega t} d t, \quad \overline{\mathrm{p}}_{v}=\int_{-\infty}^{+\infty} \mathrm{p}_{v} e^{-j \omega t} d t .
$$

From Eq. (35), one can obtain the orthogonality for the natural vibration modes.

$$
\begin{aligned}
& \int_{0}^{L} \bar{\eta}_{v}^{T}\left(\mathrm{~K} \frac{\partial^{4}}{\partial x^{4}}+\mathrm{K}_{12}\right) \bar{\eta}_{u} d x=0 \\
& \int_{0}^{L} \bar{\eta}_{v}^{T} M \bar{\eta}_{u} d x+\rho \sum_{n}\left[\begin{array}{cc}
\alpha_{n} & \beta_{n} \\
\beta_{n} & \alpha_{n}
\end{array}\right] \int_{0}^{L} \bar{\eta}_{v} \cos k_{x n} x d x \int_{0}^{L} \bar{\eta}_{u} \cos k_{x n} x d x=0
\end{aligned}
$$

Let

$$
\begin{aligned}
& \int_{0}^{L} \bar{\eta}_{u}^{T}\left(\mathrm{~K} \frac{\partial^{4}}{\partial x^{4}}+\mathrm{K}_{12}\right) \bar{\eta}_{u} d x=K_{u}^{e} \\
& \int_{0}^{L} \bar{\eta}_{u}^{T} M \bar{\eta}_{u} d x+\rho \sum_{n}\left[\begin{array}{c}
\alpha_{n} \beta_{n} \\
\beta_{n} \alpha_{n}
\end{array}\right]\left(\int_{0}^{L} \bar{\eta}_{u} \cos k_{x n} x d x\right)^{2}=M_{u}^{e},
\end{aligned}
$$

according to Eqs (33) and (36), one can obtain

$$
\bar{\eta}=\sum_{u} \frac{\bar{\eta}_{u} \bar{\eta}_{u}^{T}\left(x_{1}\right)}{K_{u}^{e}-M_{u}^{e} \omega^{2}} \mathrm{~F}
$$

Note that the displacement $\eta$ is composed of the symmetric and the anti-symmetric modes, that is $\eta=\eta_{s}+\eta_{a}$, where the subscript " $s$ " denotes the displacement composed of the symmetric modes and "a" denotes the displacement of the anti-symmetric modes, Eq. (37) implies that

$$
\bar{\eta}=\bar{\eta}_{s}+\bar{\eta}_{a}=\left(\mathrm{H}_{s}+\mathrm{H}_{a}\right) \mathrm{F} \text { or } \bar{\eta}_{s}=\mathrm{H}_{s} \mathrm{~F}, \bar{\eta}_{a}=\mathrm{H}_{a} \mathrm{~F}
$$

where $\mathrm{H}_{s}$ and $\mathrm{H}_{a}$ are the frequency response functions corresponding to the symmetric and the anti-symmetric modes, respectively. Computation of $\mathrm{H}_{a}$ and $\mathrm{H}_{s}$ are given by Eqs (17) and (14), Eqs (29), (20) and (31), respectively. Based on Eq. (37), influence of the fluid and the mechanical link to vibration transmission can be analyzed.

Impulse response functions (IRFs) are the results of the inverse Fourier transform of FRFs. In light of Eq. (38), the response to a unit impulse force can be given by

$$
\eta(t)=\frac{1}{2 \pi} \int_{-\infty}^{+\infty}\left(\mathrm{H}_{s}(\omega)+\mathrm{H}_{a}(\omega)\right) e^{j \omega t} d \omega
$$

where $\eta(t)$ is in fact composed of undamped free vibrations. However, structural damping can be taken into account by using the complex stiffness matrix $\mathrm{K}(\mathrm{I}+j \chi)$ instead of $\mathrm{K}$ in Eq. (33), and accordingly, a damped free response $\eta(t)$ can be obtained from Eq. (39). It should be noted that Eq. (39) is a simple and direct way to obtain an impulse response from the corresponding FRF, but certain errors may be generated in the impulse response due to inherent drawbacks in digital signal processing, such as energy leakage and noncausality.

\section{Transmission control}

In Fig. 1, vibration transmission from Beam-I to Beam-II is controlled by the active link. The active force $f_{c}$ is expected to generate vibrations in Beam-II to cancel the influence of the disturbance force $f_{d}$. The resultant vibration in Beam-II will decrease as the control action is well exerted, and the transmission of vibration from 


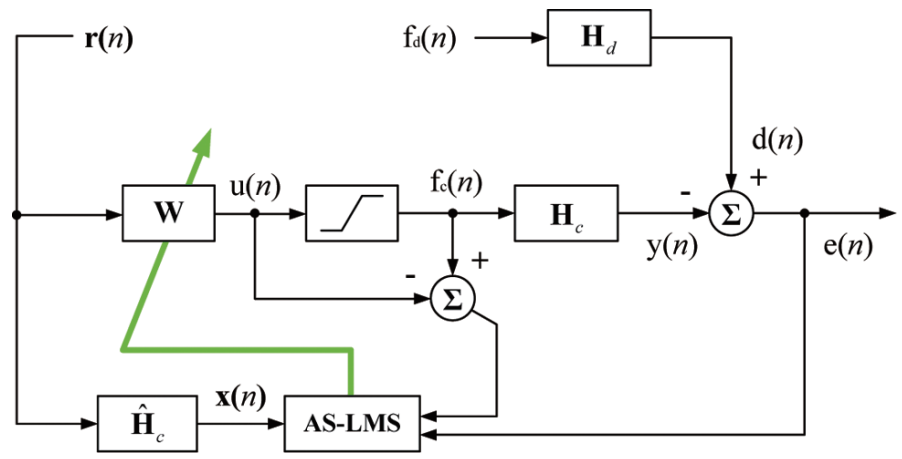

Fig. 2. Structure of an adaptive control system.

Beam-I to Beam-II will be suppressed. To carry out the active control, the vibration of Beam-II is measured by a collocated accelerometer at the center point of Beam-II, and the control force $f_{c}$ reacts according to the vibration at this location. In this study, the adaptive control system shown in Fig. 2 is adopted, where $\mathrm{H}_{c}$ is the FRF of the control channel (between the control force $f_{c}$ and the response of Point $\mathrm{C}$ ), $\hat{\mathrm{H}}_{c}$ the estimate of $\mathrm{H}_{c}, \mathrm{~W}$ the transfer function of the controller, $u(n)$ the bounded output, $e(n)$ the cancelled response, $\mathrm{r}(n)$ the reference sequence, $\mathrm{x}(n)$ the filtered reference sequence, $f_{d}(n)$ the disturbance force, $d(n)$ the response induced by $f_{d}(n), n$ the discrete time, $\mathrm{H}_{d}$ is the FRF of the disturbance channel (between the disturbance force $f_{d}$ and the response of Point C), and AS-LMS is a modified LMS algorithm [22]. $\mathrm{H}_{c}$ and $\mathrm{H}_{d}$ are computed according to Eq. (37).

The control signal is given by

$$
u(n)=g\left(\mathrm{w}^{T}(n) \mathrm{r}(n)\right)
$$

where "T" denotes the vector transpose, $g(\cdot)$ is a sigmoid function, $\mathrm{w}(n)$ is the vector of the controller coefficients. The updating formula for $\mathrm{w}(n)$ is given as

$$
\mathrm{w}(n+1)=g_{u}\left(\mathrm{w}(n)+\mu \frac{e(n) \mathrm{x}(n)}{\gamma+\mathrm{x}^{T}(n) \mathrm{x}(n)}\right)
$$

where $g_{u}$ is the derivative of $g(u)$ with respect to the variable $u, 0<\mu<1, \gamma>0$. In practice, there are many control strategies that can give optimal control results. Here, the optimal control is achieved through online adaptation of controller coefficients. Theoretically, the vibration of Point $\mathrm{C}$ will vanish after an optimal adaptive cancellation. Therefore,

$$
H_{d} F_{d}-H_{c} F_{c}=0
$$

where $F_{c}, F_{d}$ are the Fourier transform of $f_{c}$ and $f_{d}$, respectively. According to Eq. (42), the optimal control force $F_{c}$ is dependent on the disturbance force $F_{d}$. Given $F_{c}, F_{d}$, the controlled responses of the beams can be obtained from the equations given in Section 3.

In the special case that only the symmetric modes are excited and the control force cancels completely the vibration of Beam-II, the following equations should be satisfied:

$$
\left[\begin{array}{cc}
{\left[\Lambda_{1}\right]-[\sigma]+[\Theta]} & -\left(\left[\Lambda_{2}\right]+[\tau]+[\Theta]\right) \\
-\left(\left[\Lambda_{2}\right]+[\tau]+[\Theta]\right) & {\left[\Lambda_{1}\right]-[\sigma]+[\Theta]}
\end{array}\right]\left[\begin{array}{l}
\mathrm{B}_{1 c} \\
\mathrm{~B}_{2 c}
\end{array}\right]=\left[\begin{array}{c}
\mathrm{G} \\
-\mathrm{G}
\end{array}\right], \quad \mathrm{B}_{2}+\mathrm{B}_{2 c}=0
$$

where $G$ is the load vector corresponding to the control force, and $B_{2}$ satisfies Eq. (28). Equation (43) implies that

$$
\mathrm{B}_{1 c}+\mathrm{B}_{2 c}=0
$$

For the in-phase vibration excited by the disturbance force, there exists $B_{1}=B_{2}$. Therefore, in light of Eqs (43) and (44), $\mathrm{B}_{1}+\mathrm{B}_{1 c}=2 \mathrm{~B}_{1}$, but for the out-of-phase vibration excited by the disturbance force, there exists $\mathrm{B}_{1}=-\mathrm{B}_{2}$. In this case, Eqs (43) and (44) imply that $\mathrm{B}_{1}+\mathrm{B}_{1 c}=0$. Hence, the controlled vibration of Beam-I will be amplified or reduced, which depends on whether the vibration of the two beams is in-phase or out-of-phase. 
Table 1

Comparison of results by FEM and the analytical method

\begin{tabular}{lccc}
\hline \multirow{2}{*}{ Order } & \multicolumn{3}{c}{ Natural frequencies (Hz) } \\
\cline { 2 - 4 } & FEM & Analytical & Discrepancy (\%) \\
\hline 1 & 6.1449 & 6.0920 & -0.861 \\
2 & 9.9368 & 9.9368 & 0.000 \\
3 & 25.180 & 25.185 & 0.020 \\
4 & 58.683 & 55.585 & -5.279 \\
5 & 59.360 & 59.380 & 0.037 \\
6 & 84.910 & 84.944 & 0.040 \\
7 & 108.67 & 108.81 & 0.129 \\
8 & 168.40 & 168.60 & 0.119 \\
9 & 177.04 & 174.16 & -1.626 \\
10 & 244.92 & 245.28 & 0.147 \\
11 & 265.26 & 265.72 & 0.173 \\
12 & 364.89 & 365.72 & 0.227 \\
13 & 374.31 & 372.22 & -0.558 \\
\hline
\end{tabular}

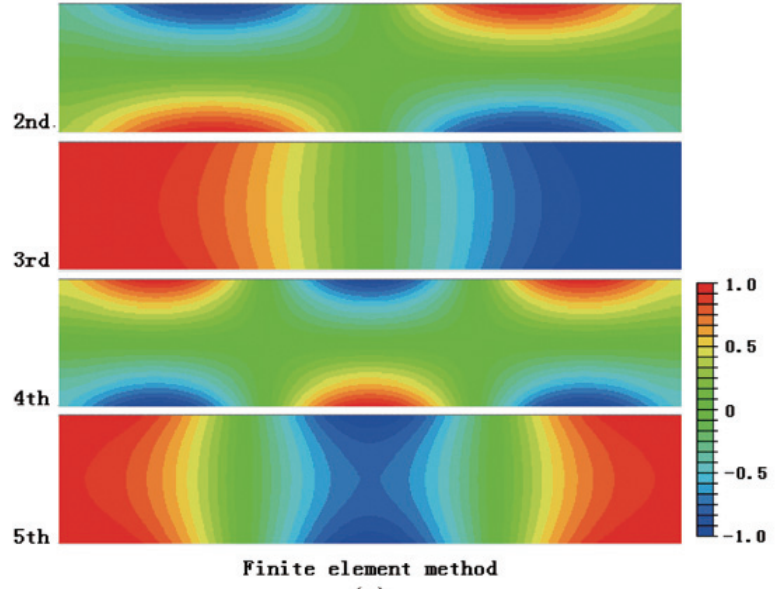

(a)

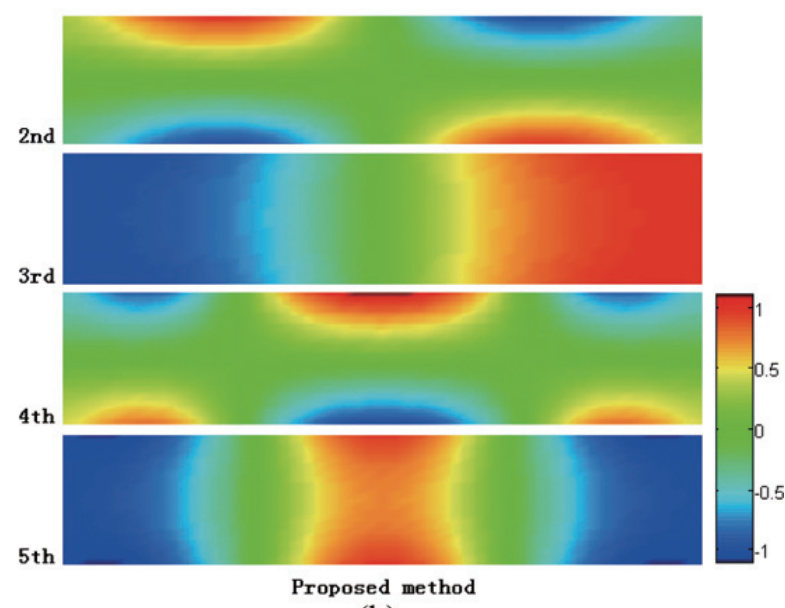

(b)

Fig. 3. Normalized pressure distribution at natural frequencies, computed with two different methods.

\section{Numerical simulation}

Considering the model shown in Fig. 1, the length $\mathrm{L}$ and the thickness $\mathrm{H}$ of the beams are $1 \mathrm{~m}$ and $0.005 \mathrm{~m}$, respectively, and the distance $2 \mathrm{~h}$ between the two beams is $0.2 \mathrm{~m}$. Mass density of the beam is $39.2 \mathrm{~kg} / \mathrm{m}$, the bending stiffness $E I=2094 \mathrm{Nm}^{2}$, mass density of the fluid $\rho=1000 \mathrm{~kg} / \mathrm{m}^{2}$, the spring stiffness $k=5 \times 10^{6} \mathrm{~N} / \mathrm{m}$, the spring and the active link is at $x_{2}=0.5 \mathrm{~L}$, and the disturbance force acts on Beam-I.

Natural frequencies of the model are listed in Table 1, from which one can see that the results given by the two methods are in good consistency. The distribution of normalized pressure is depicted in Fig. 3, from which one can also see that pressure distributions computed by the two methods are similar, and that the pressure distribution corresponding to the in-phase modes is quantitatively different from that corresponding to the out-of-phase modes.

Figure 4 shows FRFs between the disturbance force (at $x_{1}=0.27 \mathrm{~L}$ ) and the responses at three selected points on Beam-I and Beam-II, which are excited directly by the force and the active link/fluid, respectively. The depicted FRFs include all symmetric and anti-symmetric modes. It can be seen from the FRF curves in Fig. 4 that the peaks at these resonant frequencies are almost the same, and vibration transmission is not attenuated over the frequency range except at local anti-resonance frequencies. This is attributed to the fact that the two beams are strongly coupled by the fluid, which actually acts as a bridge and transmits vibrations between the two beams. When the disturbance force acts at the center point of Beam-I, only the symmetric modes can be excited. Figure 5 gives FRFs between 


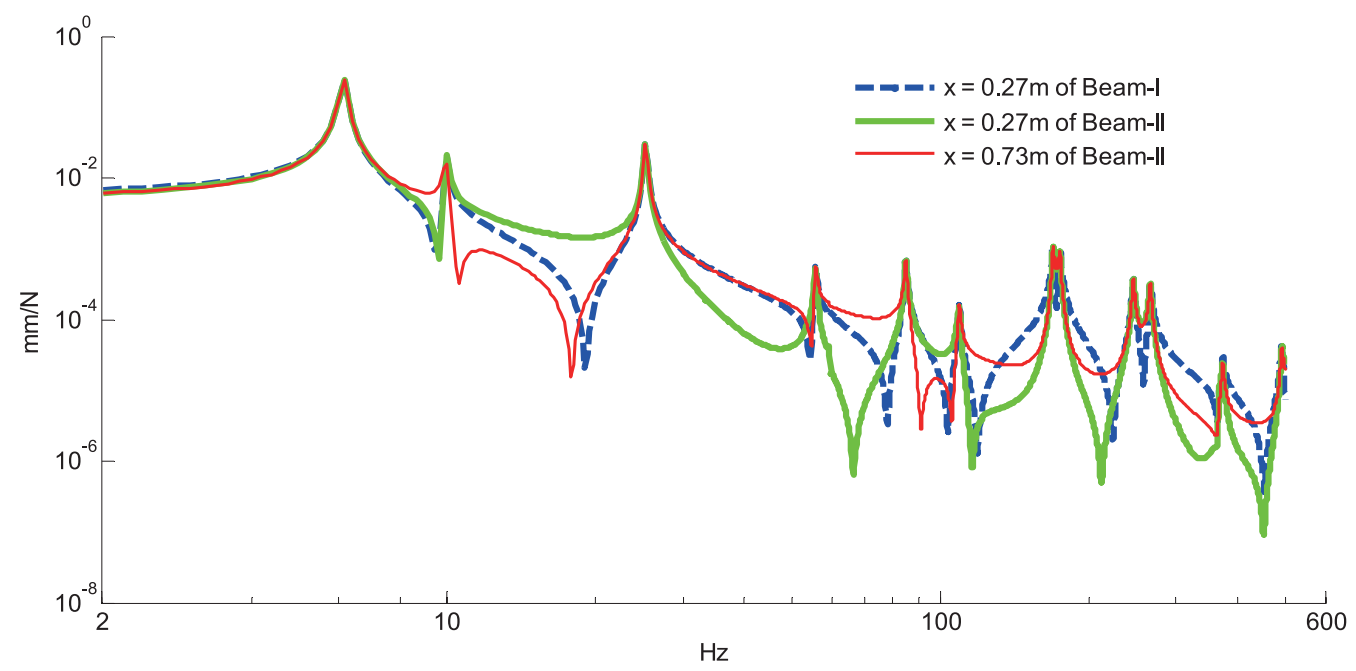

Fig. 4. Magnitude of the frequency response functions.

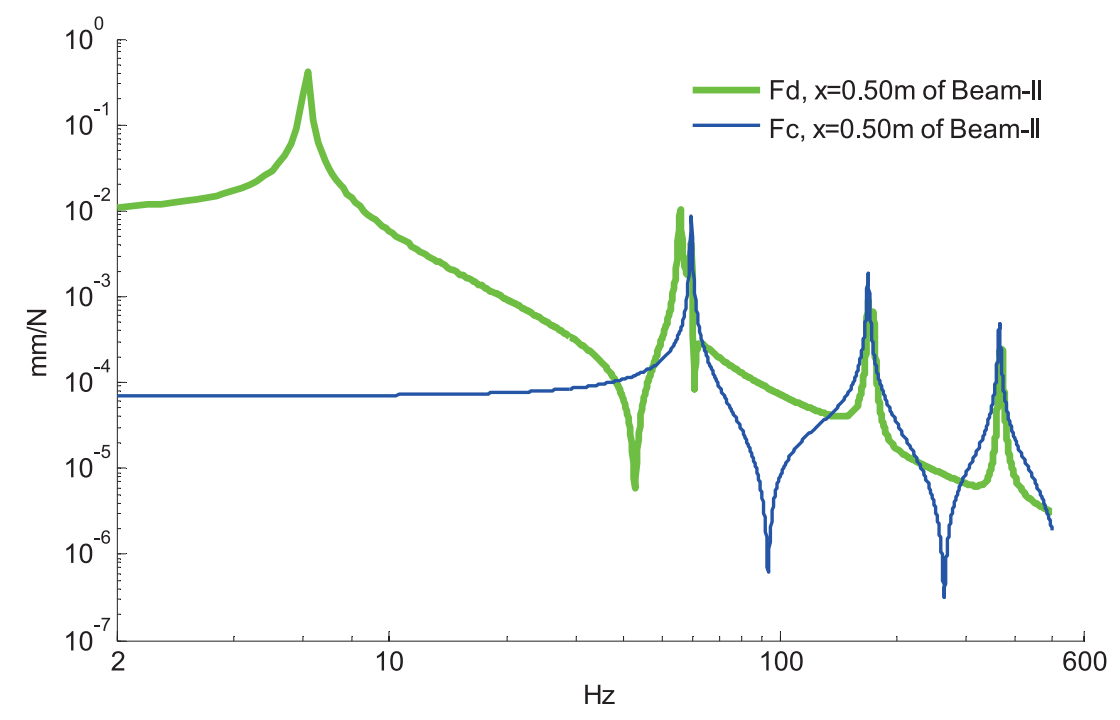

Fig. 5. Magnitude of FRFs (symmetric modes only).

the disturbance force/the control force (at $x_{1}=0.5 \mathrm{~L}$ ) and the response of the center point of Beam-II (Point C). The comparison of these FRFs computed with the proposed method and FEM is given in Fig. 6, which indicates a consistency of the two methods. In the following discussion, active control considers merely the case where excitation is at the center point of Beam-I and exhibits the involved phenomena.

Figure 7 shows the normalized natural mode shapes of the coupled beams, of which the first two are anti-symmetric and the latter two are symmetric.

The mechanical link can change natural frequencies of the coupled beams. Table 2 gives the variation of frequencies with the stiffness of the mechanical link. Since the mechanical link is at the center and no bending stiffness is considered, only the out-of-phase modes that have no nodes at the center are affected by the stiffness. According to Table 2, one can see that small stiffness is of weak influence on the natural frequencies of the coupled system, but the frequencies increase noticeably when the stiffness is large enough. The reason for this phenomenon is that the rigidity of the connected beams is affected by the stiffness of the spring. The larger the stiffness, the more rigid are the connected beams, restricting the motion of the out-of-phase modes. 


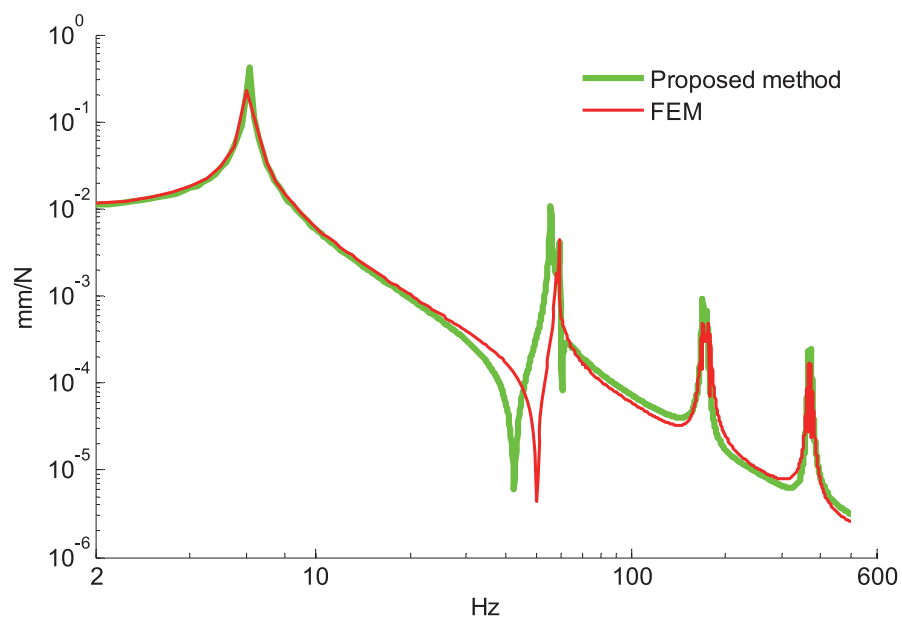

Fig. 6. Comparison of FRFs computed with different methods.

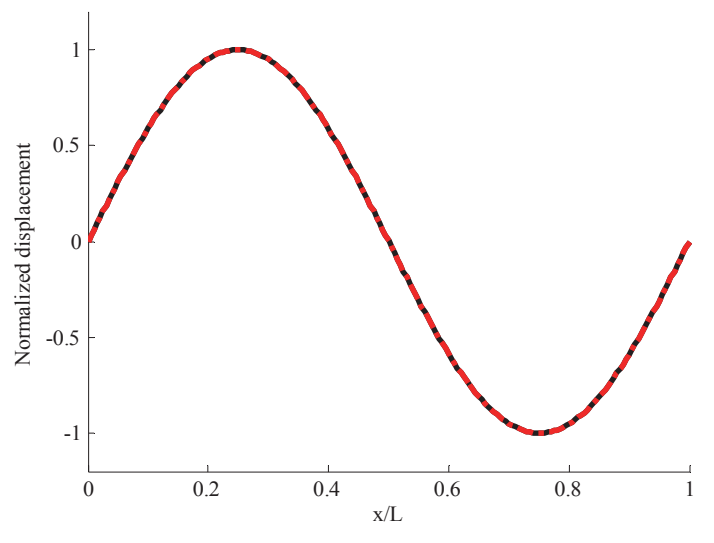

2nd $(9.9368 \mathrm{~Hz})$

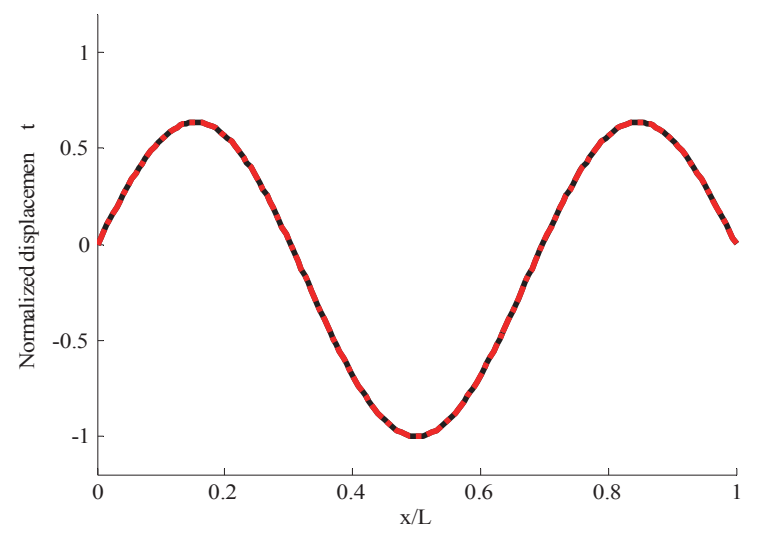

4th $(55.585 \mathrm{~Hz})$

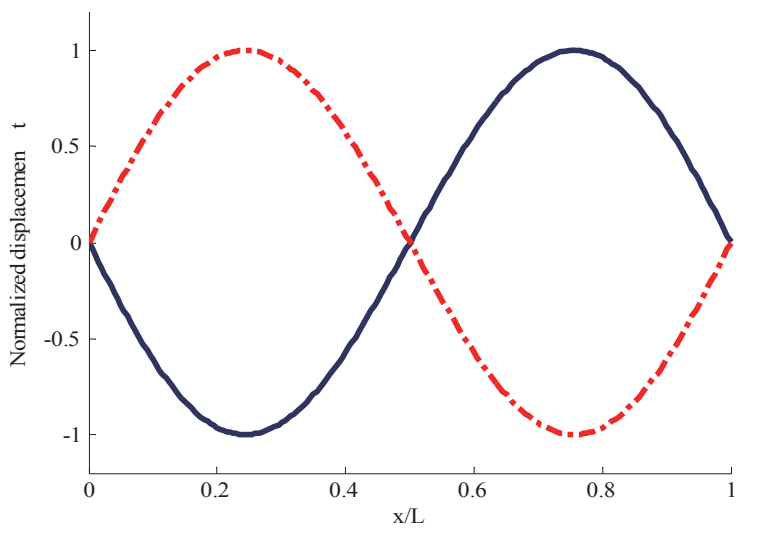

$3 \mathrm{rd}(25.185 \mathrm{~Hz})$

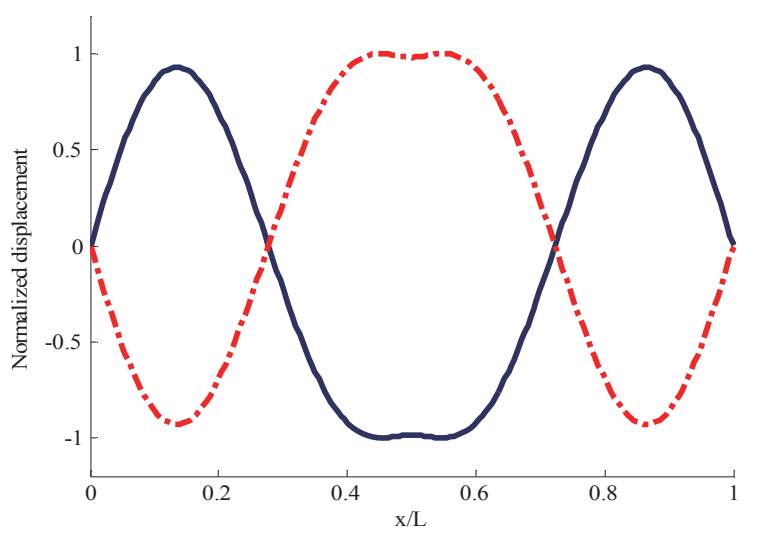

5 th $(59.380 \mathrm{~Hz})$

Solid line: displacement of Beam-I; Dash-dot line: displacement of Beam-II

Fig. 7. Selected mode shapes of the beams Solid line: displacement of Beam-I; Dash-dot line: displacement of Beam-II. 
Table 2

Variation of natural frequencies to the connecting stiffness

\begin{tabular}{lccc}
\hline & \multicolumn{3}{c}{ out-of-phase modes $(\mathrm{Hz})$} \\
\cline { 2 - 4 } Stiffness (N/m) & 2-nodes & 4-nodes & 6-nodes \\
\hline 0 & 37.05 & 154.50 & 357.40 \\
2e4 & 37.08 & 154.54 & 357.44 \\
2e5 & 38.42 & 155.10 & 357.75 \\
2e6 & 48.73 & 160.28 & 360.72 \\
5e6 & 59.38 & 168.60 & 365.72 \\
$1 \mathrm{e} 7$ & 69.32 & 181.00 & 374.08 \\
\hline
\end{tabular}

Table 3

Variation of natural frequencies to the parameter $2 \mathrm{~h} / \mathrm{L}$

\begin{tabular}{cccccc}
\hline & \multicolumn{2}{c}{$\begin{array}{c}\text { in-phase } \\
\text { modes }(\mathrm{Hz})\end{array}$} & & \multicolumn{2}{c}{$\begin{array}{c}\text { Out-of-phase } \\
\text { modes }\end{array}$} \\
\cline { 2 - 3 } \cline { 5 - 6 } 2h/L & 1-node & 2-nodes & & 1-node & 2-nodes \\
\hline 0.1 & 7.22 & 66.00 & & 30.68 & 45.55 \\
0.2 & 9.94 & 55.59 & & 25.19 & 59.38 \\
0.4 & 13.08 & 48.62 & & 20.62 & 70.36 \\
0.6 & 14.78 & 46.79 & & 18.68 & 73.42 \\
0.8 & 15.62 & 46.27 & & 17.72 & 74.42 \\
1.0 & 16.10 & 46.22 & & 17.22 & 74.58 \\
\hline
\end{tabular}

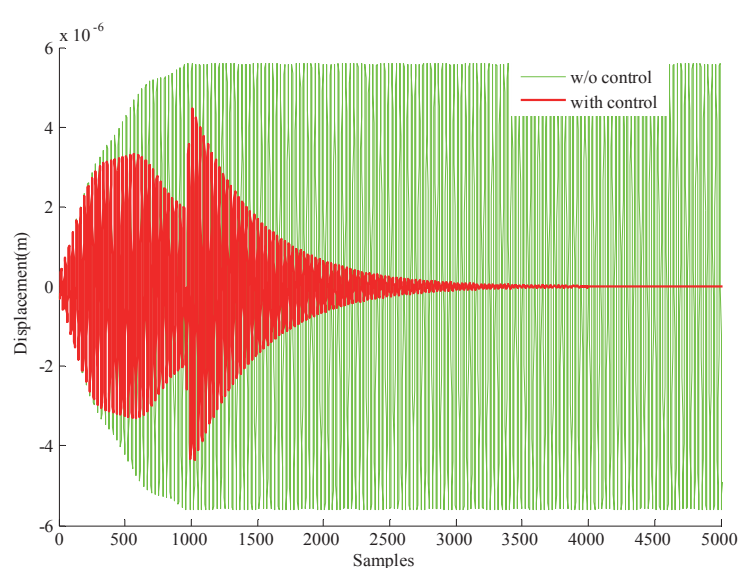

(a) Responses of Point $\mathrm{C}$ at $55 \mathrm{~Hz}$

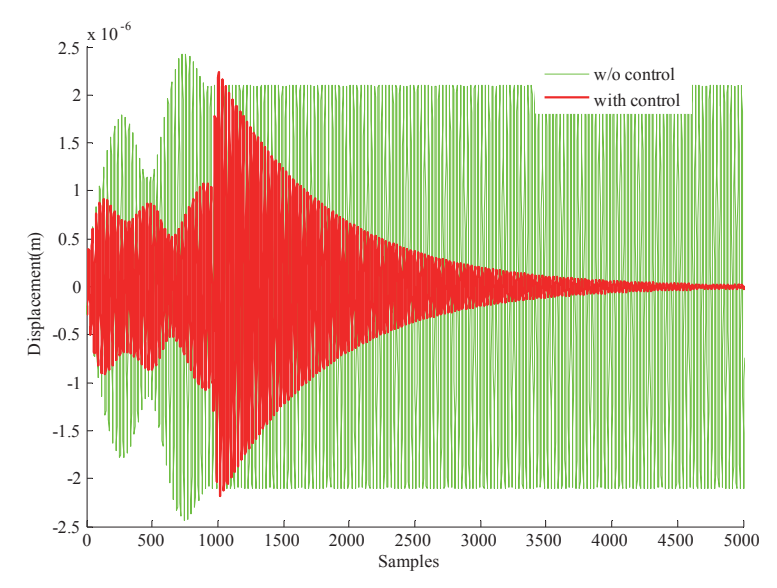

(b) Responses of Point C at $59 \mathrm{~Hz}$

Fig. 8. Controlled vibration of Beam-II.

Table 3 shows the influence of the distance between the two beams to the natural frequencies of the fluid-beam coupled system. It is interesting to see that natural frequencies of the in-phase modes decrease with the distance while those of the out-of-phase modes increase with the distance. For the in-phase modes, the inertia of the fluid is a dominant factor that reduces the frequencies of the coupled system. However, for the out-of-phase modes, compressibility of the fluid may be the factor that affects motion of the fluid from the zone of positive pressure to the zone of negative pressure, as implied in Fig. 3.

The spring between the two beams strengthens the transmission of vibration. Active control attempts to suppress the vibration of Beam-II by exerting a force on it according to a control law. As a result, coupling between the two beams is weakened and the transmission of vibration through the spring is reduced. Control force in this coupled system is generated by the active mechanical link. This force is expected to cancel the restoring force caused by the spring and further suppress the beam response induced by the sound pressure. In the simulation, considered is only the vibration at $55 \mathrm{~Hz}$ and $59 \mathrm{~Hz}$, which are approximately the 4th and 5 th natural frequencies. The reason 


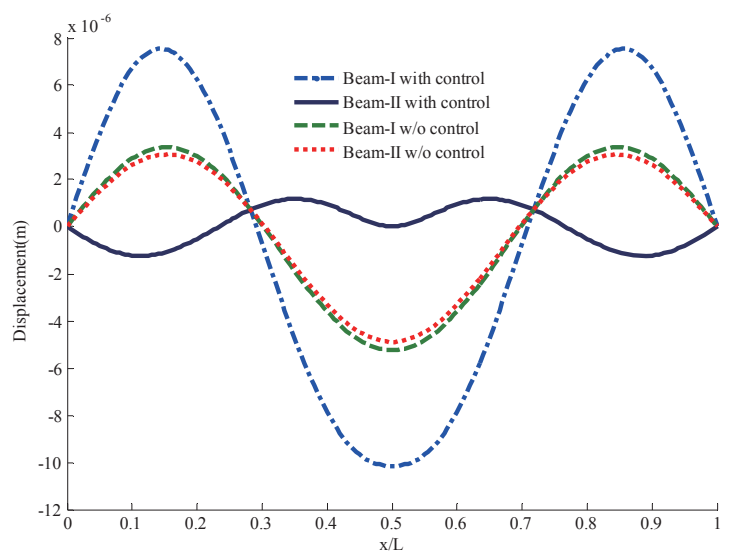

(a) At $55.0 \mathrm{~Hz}$

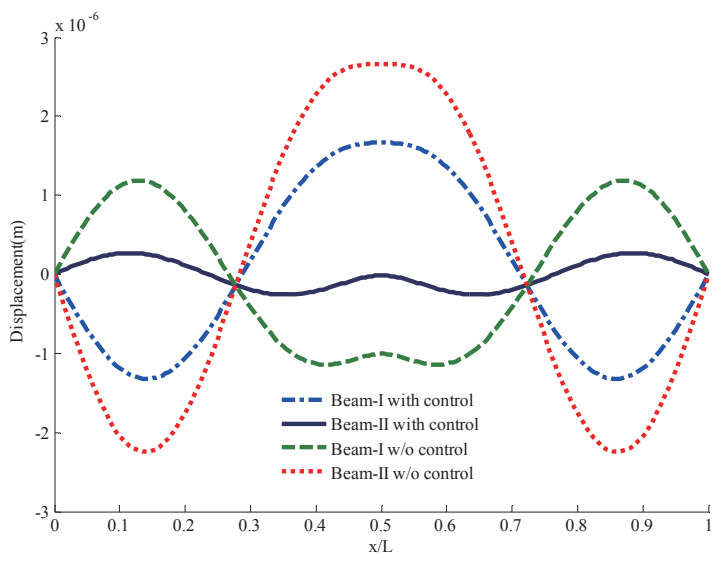

(b) At $59.0 \mathrm{~Hz}$

Fig. 9. Displacements of the coupled beams.

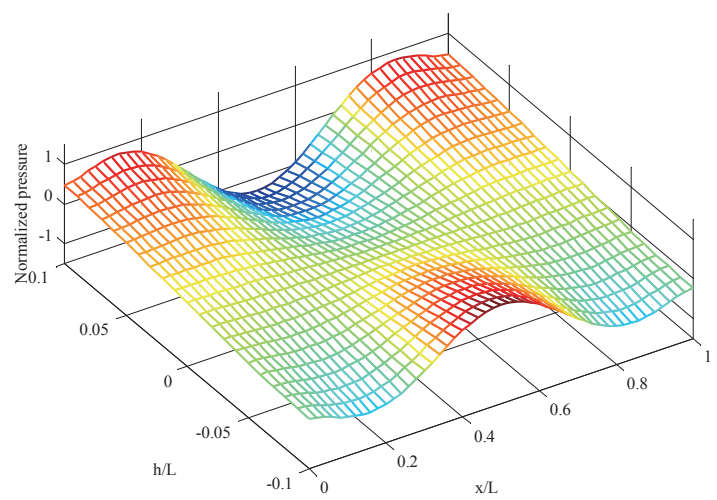

(a) Pressure distribution at $55 \mathrm{~Hz}$, without control

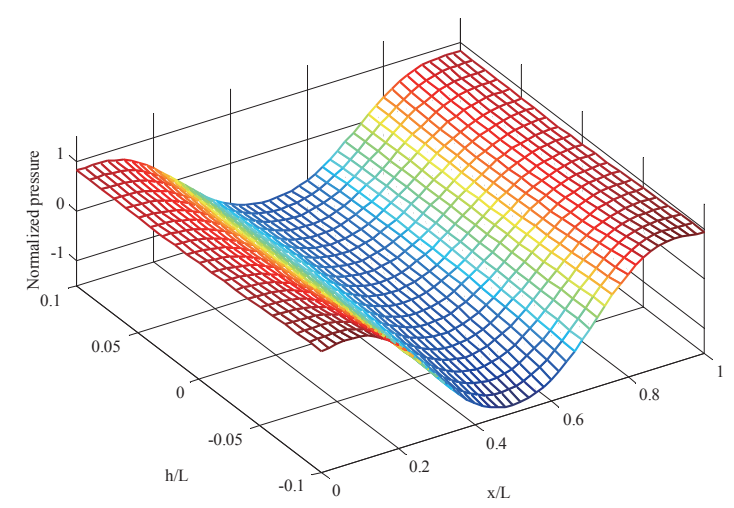

(c) Pressure distribution at 59Hz, without control

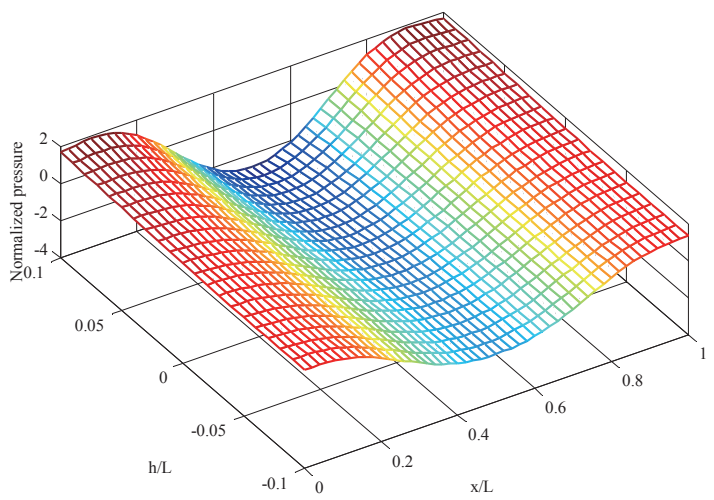

(b) Pressure distribution at $55 \mathrm{~Hz}$, with control

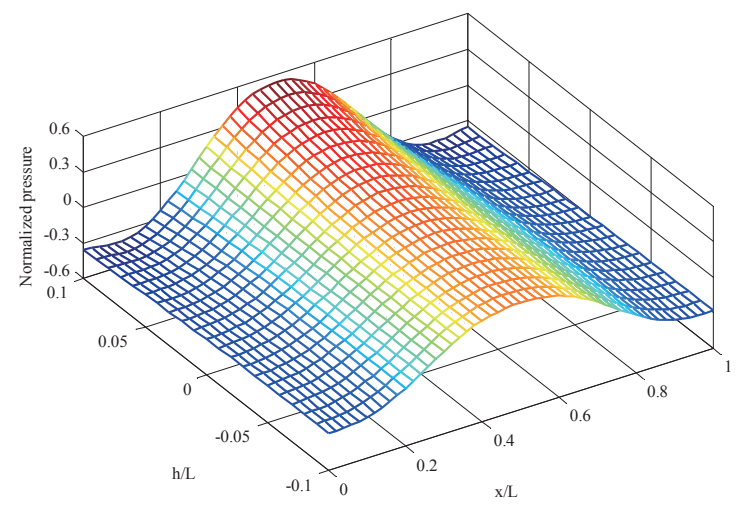

(d) Pressure distribution at $59 \mathrm{~Hz}$, with control

Fig. 10. Pressure distribution with and without control. 
for selecting these two modes in this study is that there are interesting phenomena in the coupled system when control action is turned on. In the simulation, amplitude of the disturbance force is set to $1 \mathrm{~N}$. To obtain optimal control forces, the FRFs shown in Fig. 5 are first transformed into finite impulse responses, which are used in the adaptive cancellation procedure given by Eqs (40) and (41). As the control error approaches zero, the control force also approaches its optimum. Figure 8 gives the responses of Point $\mathrm{C}$ on Beam-II before and after control. As is expected, the adaptive cancellation gives a cancelled response. When an optimal control force is obtained, the amplitude of vibration at any position is able to be computed according to the equations given in Section 3 . Vibration amplitudes of the two beams are shown in Fig. 9. It can be seen that the vibration at Point $\mathrm{C}$ nearly disappears after control. For the vibration at $55 \mathrm{~Hz}$, the displacement of Beam-II becomes very small, but that of Beam-I is amplified, which agrees well to the conclusions inferred from Eqs (43) and (44). For the vibration at $59 \mathrm{~Hz}$, displacement of Beam-II is reduced and it approaches zero. Changes in vibration modes of the beams have induced a transition of pressure distribution. Figure 10 shows the distribution of sound pressure before and after control. It can be seen from Fig. 10(a) and (b) that the pressure becomes higher after control. This is in fact caused by the increased discrepancy in vibration amplitudes and phases as shown in Fig. 9(a). Moreover, for the pressure distribution at $55 \mathrm{~Hz}$, the surface shape in Fig. 10(b) is qualitatively different from that in Fig. 10(a), which is attributed to the change in vibration modes of the beams under control (i.e. from the in-phase vibration mode to the out-of-phase vibration mode). For the pressure distribution at $59 \mathrm{~Hz}$, lower pressure can be observed in Fig. 10(d) because control action results in a reduced relative vibration in the two beams as shown in Fig. 9(b). Therefore, in both cases, interaction between the fluid and the two beams is changed by the active control.

\section{Conclusions}

Interaction between the fluid and structures has a great influence on the dynamics of structures, and for plate/shell structures with bounded fluid and mechanical links inside, vibration transmission is complicated by the fluidstructure interaction. Active elements in mechanical links have a potential in reducing vibration transmission and the mechanism is worthy of discussion. In this work, vibration characteristics of a simplified physical model, a double-beam structure with bounded fluid and active mechanical links inside, is investigated. An analytical method is presented to model the coupled system and compute its natural characteristics and vibration responses. Based on this modeling, simulation is conducted to exhibit vibration transmission between the two beams through the active element that is integrated into the structure. Simulation results have demonstrated that the active control force can change greatly the vibration modes as well as the distribution of sound pressure, and the transmission can be suppressed by canceling the vibration induced by the elastic link and the fluid. Moreover, the results given by the presented method are in good consistency with those by the finite element analysis, which implies that the modeling approach is feasible and accurate.

\section{Acknowledgements}

This work was supported in part by the State Key Laboratory of Mechanical System and Vibration (Grant No. MSV-MS- 2010-10) and the SJTU-SMC plan for excellent young teachers.

\section{References}

[1] H.H. Bleich and M.L. Baron, Free and forced vibration of an infinitely long cylindrical shell in a infinite acoustic medium, Journal of Applied Mechanics (1954)(2), 167-177.

[2] C.Y. Liaw and A.K. Chopra, Dynamics of towers surrounded by water, Earthquake Engineering and Structural Dynamics 3 (1974), 33-49.

[3] W.D.L. Finn, E. Varoglu and S. Cherry, Seismic water pressures against concrete dams, Proc. Symp. Structural and Geotechnical Mechanics, Prentice Hall (1977), 420-442.

[4] D. Zhou, Analysis of free vibration of cantilever beams in contact on both sides with fluid, Engineering Mechanics (In Chinese) 8(3) (1991), 107-115. 
[5] J.T. Xing, W.G. Price, M.J. Pomfret and L.H. Yam, Natural vibration of a beam-water interaction system, Journal of Sound and Vibration 199 (1997), 491-512.

[6] J.T. Xing, Natural vibration of two-dimensional slender structure-water interaction systems subject to Sommerfeld radiation condition, Journal of Sound and Vibration 308 (2007), 67-79.

[7] H.F. Bauer, Frequencies of a hydroelastic rectangular system, Forschung im Ingenieurwesen-Engineering Research 59 (1993), $18-28$.

[8] D. Fleischer and S.K. Park, Plane hydroelastic beam vibrations due to uniformly moving one-axle vehicle, Journal of Sound and Vibration 273 (2004), 585-606.

[9] J.S. Wu and K.W. Chen, An alternative approach to the structural motion analysis of wedge-beam offshore structures supporting a load, Ocean Engineering 30 (2003), 1791-1806.

[10] R.P.S. Han and H. Xu, A simple and accurate added mass model for hydrodynamic fluid-structure interaction analysis, Journal of the Franklin Institute 333(6) (1996), 929-945.

[11] K. Jeong, G. Yoo and S. Lee, Hydroelastic vibration of two identical rectangular plates, Journal of Sound and Vibration 272 (2004), $539-555$.

[12] M. Amabili, Ritz method and substructuring in the study of vibration with strong fluid-structure interaction, Journal of Fluids and Structures 11 (1997), 507-523.

[13] D. Zhou and Y.K. Cheung, Vibration of vertical rectangular plate in contact with water on one side, Earthquake Engineering and Structural Dynamics 29 (2000), 693-710.

[14] L. Cheng, Y.Y. Li and J.X. Gao, Energy transmission in a mechanically-linked double-wall structure coupled to an acoustic enclosure, Journal of Acoustical Society of America 117(5) (2005), 2742-2751.

[15] F.X. Xin, T.J. Lu and C.Q. Chen, Sound transmission through simply supported finite double-panel partitions with enclosed air cavity, Journal of Vibration and Acoustic 132 (2010), 1-11.

[16] N. Alujević, K.D. Frampton and P. Gardonio, Smart double panel with decentralised active dampers for sound transmission control, AIAA Journal 46(6) (2008), 1463-1475.

[17] S. Daley, F.A. Johnson, J.B. Pearson and R. Dixon, Active vibration control for marine applications, Control Engineering Practice (2004) (12), 465-474.

[18] S.J. Elliott, I.M. Stothers and P.A. Nelson, A multiple error LMS algorithm and its application to the active control of sound and vibration, IEEE Transactions on Acoustics, Speech, and Signal Processing 35(10) (1987), 1423-1434.

[19] E. Bianchi, P. Gardonio and S.J. Elliott, Smart panel with multiple decentralized units for the control of sound transmission. Part III: control system implementation, Journal of Sound and Vibration 274 (2004), 215-232.

[20] A.J. Hillis, A.J.L. Harrison and D.P. Stoten, A comparison of two adaptive algorithms for the control of active engine mounts, Journal of Sound and Vibration 286 (2005), 37-54

[21] F. Fahy and P. Gardonio, Sound and structural vibration (Second Edition), Academic Press Inc., 2007.

[22] Z. Zhang, F. Hu and J. Wang, On saturation suppression in adaptive vibration control, Journal of Sound and Vibration 329 (2010), 1209-1214. 

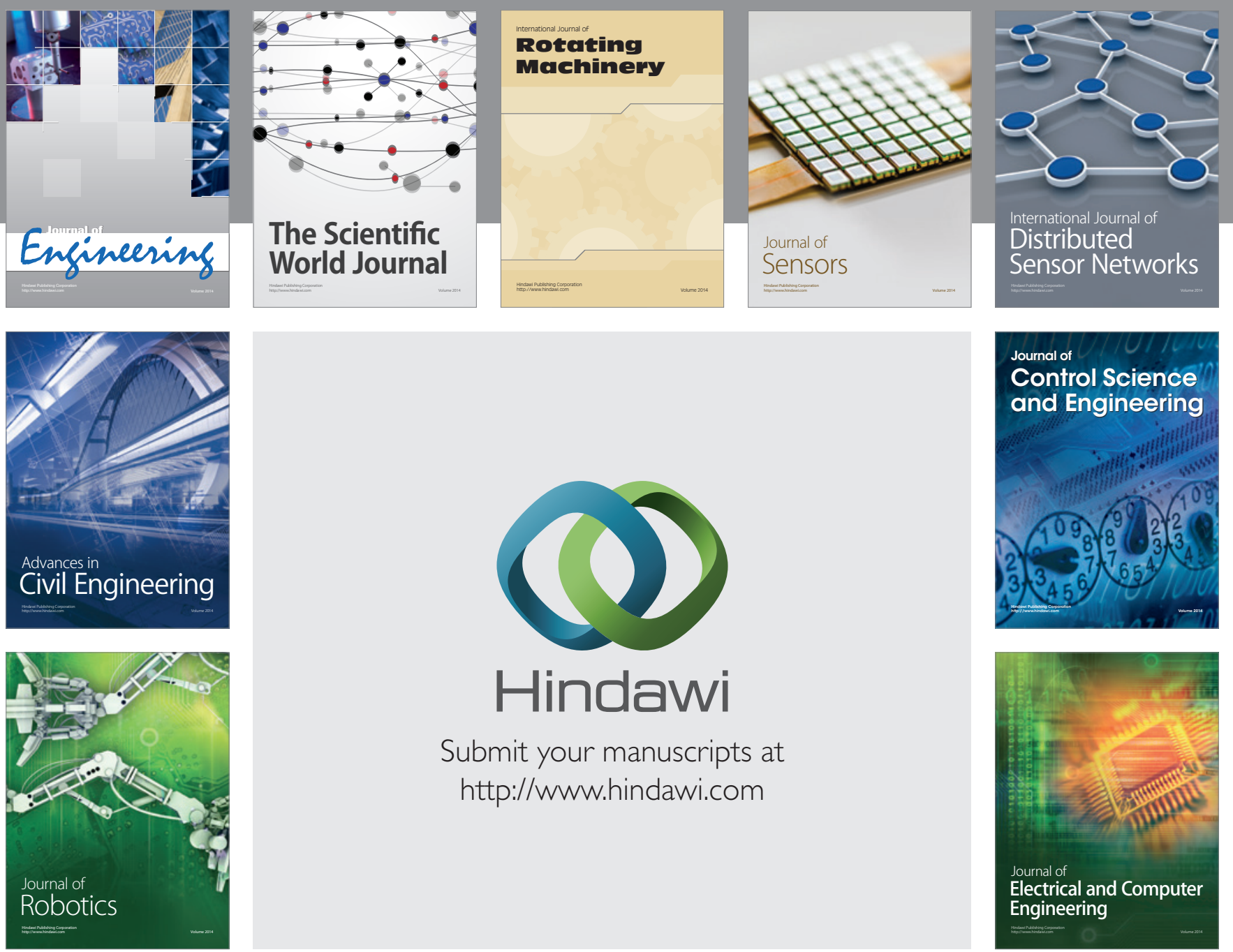

Submit your manuscripts at

http://www.hindawi.com
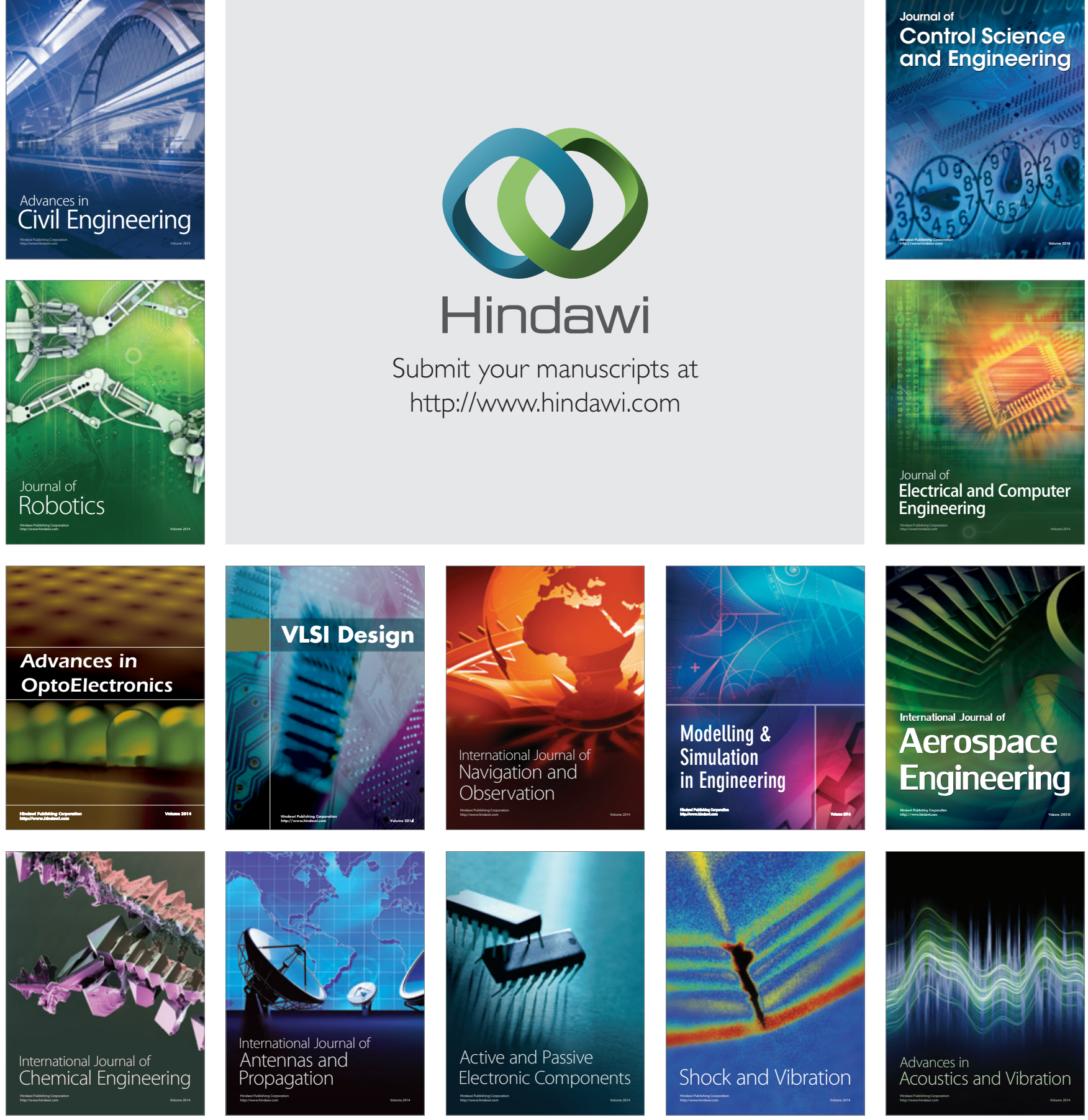